\title{
Improving the Validity of Squeeze Film Air-Damping Model of MEMS Devices with Border Effect
}

\author{
Cheng Bai ${ }^{1,2}$ and Jin Huang ${ }^{1,3}$ \\ ${ }^{1}$ Research Institute on Mechatronics, Xidian University, Xi'an 710071, China \\ ${ }^{2}$ Xian Communication Institute, Xian 710106, China \\ ${ }^{3}$ Key Laboratory of Electronic Equipment Structure Design, Xidian University, Ministry of Education, Xian 710071, China \\ Correspondence should be addressed to Jin Huang; jhuang@mail.xidian.edu.cn
}

Received 21 February 2014; Revised 6 May 2014; Accepted 14 May 2014; Published 26 May 2014

Academic Editor: Ricardo Perera

Copyright (C) 2014 C. Bai and J. Huang. This is an open access article distributed under the Creative Commons Attribution License, which permits unrestricted use, distribution, and reproduction in any medium, provided the original work is properly cited.

Evaluation of squeezed film air damping is critical in the design and control of dynamic MEMS devices. The published squeezed film air damping models are generally derived from the analytical solutions of Reynolds equation or its other modified forms under the supposition of trivial pressure boundary conditions on the peripheral borders. These treatments ignoring the border effect can not give faithful result for structure with smaller air venting gap or the double-gimbaled structure in which the inner frame and outer one affect the air venting. In this paper, we use Green's function to solve the nonlinear Reynolds equation with inhomogeneous boundary conditions. For two typical normal motion cases of parallel plate, the analytical models of squeeze film damping force with border effect are established. The viscous and inertial losses with real values and image values acoustic impedance are all included in the model. These models reduced the time consumption while giving satisfactory result. Without multifield coupling analysis, the estimation of the dynamic behavior of MEMS device is also allowed, and the simulation of the system performance is more convenient.

\section{Introduction}

With the reduction in feature size of the micromachined structures, the performances of MEMS devices are affected by air damping more significantly than machines of conventional dimensions. As long as the ratio of air gap thickness to the plate width is less than $1 / 3$, the squeeze film air damping will be greater than the drag force damping and become the main contribution to air damping [1]. Most of the micromachined sensors and actuators, such as microaccelerometers, optical switches, and microgyroscope, often have a narrow air gap. Therefore, the squeezed film damping (SFD) has important influence on the dynamic behaviors of these devices [1], and evaluation of SFD plays an important role in the design and control of dynamic MEMS devices.

According to the law of conservation of mass, momentum, and energy, the basic equations of fluid mechanics, including Euler equation, Navier-Stokes equation, and Burnett equation, are established. Because these equations are hard to be solved analytically, they are usually computed numerically to simulate the device behavior as part of a full system analysis. For complex microstructures, 3D NavierStokes-based FEM simulations requires more than a dozen hours or even a day of computing time, and the computational effort often becomes prohibitive [2]. Therefore, in order to design and control MEMS devices more efficiently, an analytical SFD model is desirable to be derived.

The SFD models are generally derived from the analytical solutions of Reynolds equation or its other modified forms under a certain boundary conditions. Andrews et al. [3] have studied the squeeze film theoretical predictions for two isolated rectangular plates oscillating normal to each other. Analytic squeeze film air damping models for a rectangular torsion mirror plate have been developed by Pan et al. [4] and Minikes et al. [5]. In all these studies, the Reynolds equation is solved with trivial pressure boundary condition $(P=0)$ on the free edges and trivial flow conditions $(V=0)$ on the fixed edges. Various models have been presented for the squeeze film analysis of perforated MEMS structures in the literature spanning over a decade; trivial venting boundary 
condition $(P=0)$ is also used $[6,7]$. In practice, moreover, the trivial boundary condition assumed that the gas pressure disappears abruptly on the border of the movable plate which does not always hold. In structure with smaller air venting gap and the double-gimbaled structure where the inner frame and outer one affect the air venting, these methods fail to give satisfactory results.

In order to model the squeeze film damping considering the border effects, Hao et al. [8] located the trivial pressure boundary in the sidewall of the mirror cavity rather than at the edge of the movable plate; moreover, if the sidewall is placed closer to the movable plate, their idea is not a good one. Compared with FEM simulations, Veijola et al. [9] derived the SFD models with effective extended dimensions. Although their method improved the accuracy of the damping calculation, it is invalid for structures with complex borders such as smaller air venting gap structure and the doublegimbaled MEMS torsion mirror. Darling et al. [10] presented their model for reactive acoustic impedance. For free plate under ideally venting conditions, the analytical model they derived is in agreement with other literature [3]. However, the validity of their model under slot venting conditions can be further extended to include viscous losses with realvalues acoustic impedance. Veijola et al. [11] focused on the nontrivial boundary conditions for the flow, accounting for the finite acoustic impedance of the surrounding gas at the borders and extended the validity of the model presented by Darling et al.

In this paper, a more consistent and intuitive approach to the solution of inhomogeneous boundary-value problems was provided. The Green's function method to the linearized Reynolds equation with inhomogeneous boundary conditions was adopted. Based on the normal motion of a rectangular parallel plate with constant velocity and oscillating velocity, novel squeeze film air damping models considering border effect are obtained. The viscous and inertial losses with real-values and image-values acoustic impedance are all included in the model.

\section{Analytical Model and Green's Function Solution}

A general parallel plates MEMS device consists of a movable plate and a stationary substrate as is shown in Figure 1; the movable plate is suspended by four micro beams. In microaccelerometers, the moving direction of the movable plate is normal to the fixed plate. The length of the movable plate is denoted by $a$, and the width is represented by $b ; H_{0}$ is the initial squeeze film thickness under the movable plate; $\delta$ is the gas venting gap between the movable plate and the cavity sidewall.

The air damping comes from the pressure distribution on the movable plate surface. Under the isothermal condition, the air pressure distribution can be modeled with the nonlinear isothermal Reynolds equation:

$$
\nabla \cdot\left(h^{3} p \nabla p\right)=\sigma \frac{\partial}{\partial t}(p h)
$$

In order to perform analytical modeling analysis, assuming variations of local pressure and squeeze film thickness are small, $p=P_{a}+\delta p, h=H_{0}+\delta h$, (1) can be linearized into

$$
\nabla^{2} P-\frac{12 \mu}{P_{a} H_{0}^{2}} \frac{\partial P}{\partial t}=\frac{12 \mu}{P_{a} H_{0}^{2}} \frac{\partial H}{\partial t},
$$

where $P$ and $H$ are the normalized pressure and the normalized squeeze film thickness, respectively, with $P_{a}$ denoting the ambient pressure.

Because this partial differential equation has the same format as the heat diffusion equation, we can refer to the solution of heat diffusion equation to solve it. In this section, we use Green's function method to solve (2). The heat diffusion equation satisfied by Green's function $(G)$ involves an impulsive point source which is

$$
\nabla^{2} G-\alpha^{2} \frac{\partial G}{\partial t}=-4 \pi \delta\left(r-r_{0}\right) \delta\left(t-t_{0}\right) .
$$

The linearized Reynolds equation (2) can be seen as a heat diffusion equation, and the heat source is a displacement function of time as $12 \mu / P_{a} H_{0}^{2} \cdot \partial H / \partial t$, where $\alpha^{2}=12 \mu / P_{a} H_{0}^{2}$ is a constant, with $\mu$ representing the air viscosity.

The solution of the inhomogeneous heat diffusion equation, with inhomogeneous boundary conditions and zero initial condition is expressible as [11]

$$
\begin{aligned}
P(r, t)= & \int_{0}^{t^{+}} d t^{\prime} \int d V^{\prime} G\left(r, t \mid r^{\prime}, t^{\prime}\right) \rho\left(r^{\prime}, t^{\prime}\right) \\
& +\frac{1}{4 \pi} \int_{0}^{t^{+}} d t^{\prime} \int d S^{\prime} \cdot\left[\operatorname{Ggrad}^{\prime} P-P \operatorname{grad}^{\prime} G\right],
\end{aligned}
$$

where $\rho\left(r^{\prime}, t^{\prime}\right)$ is the effective source point,

$$
\rho\left(r^{\prime}, t^{\prime}\right)=-\frac{\alpha^{2} \eta}{4 \pi} \frac{\partial H}{\partial t^{\prime}}
$$

and Green's function $G\left(r, t \mid r^{\prime}, t^{\prime}\right)$ can be expanded in terms of eigenfunctions [11],

$$
\begin{aligned}
& G\left(r, t \mid r^{\prime}, t^{\prime}\right) \\
& \quad=\frac{4 \pi}{\alpha^{2}} u\left(t-t^{\prime}\right) \sum_{m, n} e^{-\frac{\lambda_{m n}^{2}\left(t-t^{\prime}\right)}{\alpha^{2}}} u_{m n}(r) u_{m n}{ }^{*}\left(r^{\prime}\right) .
\end{aligned}
$$

In (6), eigenfunction $u_{m n}$ is a solution of a scalar Helmholtz equation in a region bounded by a surface $S$. Consider

$$
u_{m n}(x, y)=\frac{2}{\sqrt{a b}}_{\sin }^{\cos }\left(\frac{m \pi x}{a}\right){ }_{\sin }^{\cos }\left(\frac{n \pi y}{b}\right) .
$$

The eigenvalue $\lambda_{m n}$ is

$$
\lambda_{m n}^{2}=\lambda_{m}^{2}+\lambda_{n}^{2}=\frac{m^{2} \pi^{2}}{a^{2}}+\frac{n^{2} \pi^{2}}{b^{2}} .
$$

If $m, n$ are odd indices, cos is chosed, and sin is chosed for even indices. 


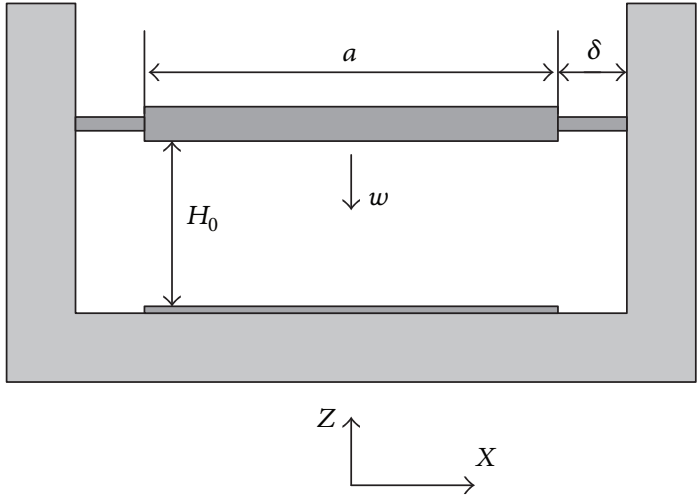

(a) Cross-section view

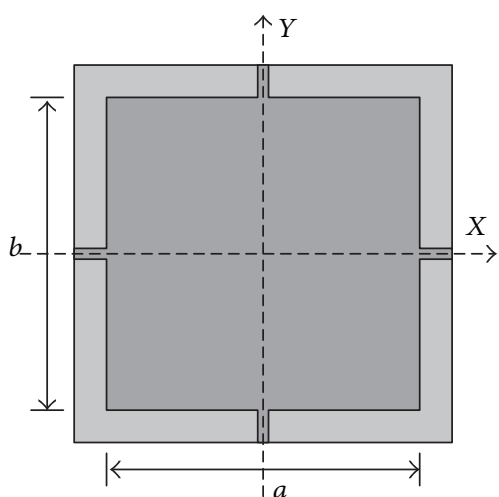

(b) Top view

FIGURE 1: Schematic diagram of a normal moving MEMS device.

As Green's function satisfies the homogeneous boundary condition, the following equation was established:

$$
\frac{1}{4 \pi} \int_{0}^{t^{+}} d t^{\prime} \int d S^{\prime} \cdot \operatorname{Ggrad}^{\prime} P=0
$$

The solution of heat diffusion equation (4) satisfies the inhomogeneous boundary condition. It means that at the edge of the movable plate, $(X, Y, t) \in S$, the solution of this equation $P(X, Y, t)$ equals to the boundary condition $\phi(X, Y, t)$. Then the solution of the inhomogeneous diffusion equation, with inhomogeneous boundary conditions, can be reduced as

$$
\begin{aligned}
P(r, t)= & \int_{0}^{t^{+}} d t^{\prime} \int d V^{\prime} \rho\left(r^{\prime}, t^{\prime}\right) G\left(r, t \mid r^{\prime}, t^{\prime}\right) \\
& -\frac{1}{4 \pi} \int_{0}^{t^{+}} d t^{\prime} \int d S^{\prime} \phi \frac{\partial G}{\partial n}
\end{aligned}
$$

\section{Normal Motion with Constant Velocity}

3.1. Damping Force. To illustrate the solution of the normal motion of a rectangular plate, the movable plate is assumed moving toward the fixed plate with a velocity of $w_{M}$. The deviatory squeeze film thickness is same as the displacement of normal motion, $\Delta h=w_{M} t$. Under this assumption, the linearized Reynolds equation has the same form with (2), where

$$
\frac{\partial H}{\partial t}=\frac{\partial\left(1+\Delta h / H_{0}\right)}{\partial t}=\frac{w_{M}}{H_{0}} .
$$

With inhomogeneous boundary conditions, the pressures of boundary are not trivial which are given by

$$
\phi=P\left( \pm \frac{a}{2}, Y, t\right)=P\left(X, \pm \frac{b}{2}, t\right)=P_{\mathrm{BC}} .
$$

The first term of (10) represents the effect of volume sources. The pressure distribution of the movable plate is

$$
\begin{aligned}
P_{1}= & \int_{0}^{t^{+}} d t^{\prime} \int d V^{\prime} \rho\left(r^{\prime}, t^{\prime}\right) G\left(r, t \mid r^{\prime}, t^{\prime}\right) \\
= & \sum_{m, n=\text { odd }} \frac{16(-1)^{(m+n) / 2}}{m n \pi^{2}} \cdot \frac{\alpha^{2}}{\lambda_{m n}^{2}} \cdot \frac{\eta w_{M}}{h_{0}}\left[1-\exp \left(\frac{-\lambda_{m n}^{2} t}{\alpha^{2}}\right)\right] \\
& \quad \times \cos \frac{m \pi x}{a} \cos \frac{n \pi y}{b} .
\end{aligned}
$$

The second term of (10) represents the effect of inhomogeneous boundary conditions; the pressure distribution of the movable plate is

$$
\begin{aligned}
P_{2}= & -\frac{1}{4 \pi} \int_{0}^{t^{+}} d t^{\prime} \int d S^{\prime} \phi \frac{\partial G}{\partial n^{\prime}} \\
= & \sum_{m, n=\mathrm{odd}} P_{\mathrm{BC}} \cdot \frac{16}{m n \pi^{2}}\left[1-\exp \left(\frac{-\lambda_{m n}^{2} t}{\alpha^{2}}\right)\right] \cdot(-1)^{(m+n) / 2} \\
& \cdot \cos \frac{m \pi x}{a} \cos \frac{n \pi y}{b} .
\end{aligned}
$$

As a consequence, the total pressure distribution of the movable plate is

$$
\begin{aligned}
& P(x, y, t) \\
& =\sum_{m, n=\text { odd }} \frac{16(-1)^{(m+n) / 2}}{m n \pi^{2}} \cdot \frac{\alpha^{2}}{\lambda_{m n}^{2}} \cdot \frac{\eta w_{M}}{h_{0}}\left[1-\exp \left(\frac{-\lambda_{m n}^{2} t}{\alpha^{2}}\right)\right] \\
& \quad \times \cos \frac{m \pi x}{a} \cos \frac{n \pi y}{b} \\
& +\sum_{m, n=\text { odd }} P_{\mathrm{BC}} \cdot \frac{16}{m n \pi^{2}}\left[1-\exp \left(\frac{-\lambda_{m n}^{2} t}{\alpha^{2}}\right)\right] \cdot(-1)^{(m+n) / 2} \\
& \cdot \cos \frac{m \pi x}{a} \cos \frac{n \pi y}{b} .
\end{aligned}
$$


The resultant force is the integration of the pressure variation across the rectangular plate area:

$$
\begin{aligned}
F(t)= & P_{a} \int_{-a / 2}^{a / 2} \int_{-b / 2}^{b / 2} P(x, y, t) d x d y \\
= & \sum_{m, n=\text { odd }} \frac{64 a b P_{a}}{m^{2} n^{2} \pi^{4}} \cdot \frac{\eta w_{M}}{h_{0}} \cdot \frac{\alpha^{2}}{\lambda_{m n}^{2}}\left[1-\exp \left(\frac{-\lambda_{m n}^{2} t}{\alpha^{2}}\right)\right] \\
& +\sum_{m, n=\text { odd }} P_{\mathrm{BC}} \frac{64 a b P_{a}}{m^{2} n^{2} \pi^{4}}\left[1-\exp \left(\frac{-\lambda_{m n}^{2} t}{\alpha^{2}}\right)\right],
\end{aligned}
$$

where $\alpha^{2}=12 \mu / \eta h_{0}^{2} P_{a}$.

In order to achieve steady state, the turn-on transient term is discarded, and then the resultant force is simplified as

$$
F(t)=\sum_{m, n=\text { odd }} \frac{64 a b P_{a}}{m^{2} n^{2} \pi^{4}} \cdot \frac{\eta w_{M}}{h_{0}} \cdot \frac{\alpha^{2}}{\lambda_{m n}^{2}}+\sum_{m, n=\text { odd }} P_{\mathrm{BC}} \frac{64 a b P_{a}}{m^{2} n^{2} \pi^{4}} .
$$

The resultant force comes from the damping pressure profile across the lower surface of the movable plate. Equation (17) expressing the reaction force in normal motion is composed by two different effect parts: the term due to the effect of volume sources and that due to the border effect.

3.2. Boundary Pressure. In the derived damping force model, the border effect is denoted as a function of the boundary pressure $P_{B C}$. To calculate the damping force with border effect, the boundary pressure should be determined firstly.

As can be seen in Figure 2, the gas venting gap between the movable plate and the sidewall can be regarded as a slot between two parallel plates. One of these two parallel plates is fixed, and the other is in vertical motion at speed $w_{M}$ in the inverse $z$-direction. The cross section length, cross section width, and channel length of the slot are $2(L+W), \delta$, and $D_{p}$, respectively. The steady flow velocity of fluid between paralleled plates is $u=v=0, w$. According to the continuity equation, $\partial u / \partial x=0$ and $\partial v / \partial y=0$. For incompressible viscous flow, ignoring the mass force, the N-S equation can be simplified as [12]

$$
\begin{gathered}
0=-\frac{\partial p}{\partial x}, \\
0=-\frac{\partial p}{\partial y}, \\
-\frac{1}{\rho} \frac{\partial p}{\partial z}+\mu \frac{\partial^{2} w}{\partial x^{2}}=0
\end{gathered}
$$

where the boundary conditions are $w=w_{M}$ at $x=0$ and $w=0$ at $x=\delta$.

By solving the above equations, the volume flow rate $Q$ between the parallel plates channel can be obtained as

$$
Q=\int_{0}^{\delta} w \cdot 2(a+b) d x=\frac{(a+b) \delta^{3}}{6 \mu} \frac{\Delta p}{D_{P}}-(a+b) \delta w_{M} .
$$

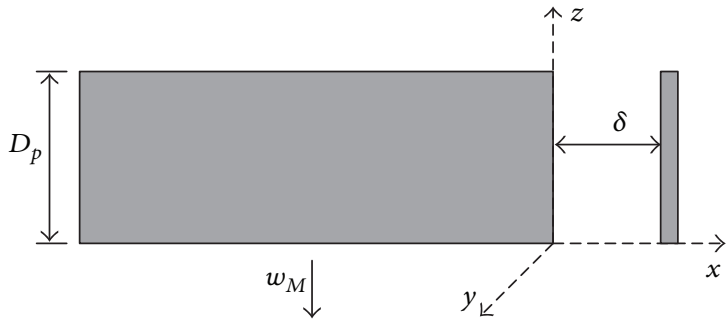

FIGURE 2: Flow between parallel plates.

The volume flow rate $Q$ between the parallel plates channel is composed of two parts: Couette flow and Poiseuille flow. The Couette flow is driven by the motion of the movable plate, and the Poiseuille flow is driven by the pressure gradient through two stationary flat walls.

Because the gas is considered as incompressible, the venting volume of gas through the slot is equal to the reduction of volume under the movable plate caused by the plate movement; the boundary pressure at the edge of the movable plant is

$$
P_{\mathrm{BC}}=\frac{6 \mu D_{P} w_{M}(a b+a \delta+b \delta)}{(a+b) \delta^{3}},
$$

where the constant velocity of the plate is $w_{M}$.

3.3. Comparison of Numerical and Simulation Results. Threedimensional computational fluid dynamics (CFD) simulations have been carried out to compare the model considering the border effects with the ideally vented model. The fluid field analysis software ANSYS/FLOTRAN has been adopted to compute the incompressible Navier-Stokes equations. Due to the symmetry, a quarter of the fluid domain was studied. In order to simulate the devices to fully open to ambient pressure, the boundaries dimensions of the fluid domain above the plate are extended by $L / 2$. Software ANSYS generated the 3D mesh, made by tetrahedral elements for the surrounding air of the movable plate. In order to improve the accuracy and efficiency of simulation, the grid below the movable plate is much denser than that above the movable plate. Figure 3(a) shows a typical mesh generation, and Figure 3(b) is a corresponding typical pressure distribution.

Serious simulations were performed with different structure dimensions. Table 1 is the comparison of squeeze film damping force in constant velocity normal motion between analytical models and the simulation results. As can be seen, the model considering the border effects is more accurate than the ideally vented model. With the gap $(\delta)$ between the movable plate and the sidewall larger than the initial squeeze film thickness $\left(H_{0}\right)$, the damping forces are close to the numerical simulation results. Whereas, with the gap $\delta$ comparable to the thickness $h_{0}$, the gas will be compressed and the model is not accurate enough. The error rate of our model is $23.55 \%$, and the error rate of ideally vented model is even $45.84 \%$. 
TABLE 1: Comparison of squeeze film damping between analytical models and simulation results in normal motion.

\begin{tabular}{|c|c|c|c|c|c|c|c|c|c|}
\hline \multirow{2}{*}{ Case } & \multicolumn{3}{|c|}{ Dimension $10^{-6} \mathrm{~m}$} & \multirow{2}{*}{ Simulation Force N } & \multicolumn{2}{|c|}{ Ideally vented model } & \multicolumn{3}{|c|}{ Our model } \\
\hline & $a, b$ & $H_{0}$ & $\delta$ & & Force N & Error rate & $P_{\mathrm{BC}}$ & Force N & Error rate \\
\hline 1 & 400 & 40 & 60 & $3.5447 e-8$ & $2.9909 e-8$ & $15.62 \%$ & 0.026 & $3.4046 e-8$ & $3.95 \%$ \\
\hline 2 & 400 & 40 & 50 & $4.1837 e-8$ & $2.9909 e-8$ & $28.51 \%$ & 0.043 & $3.6601 e-8$ & $1.63 \%$ \\
\hline 3 & 400 & 40 & 40 & $5.5220 e-8$ & $2.9909 e-8$ & $45.84 \%$ & 0.081 & $4.2217 e-8$ & $23.55 \%$ \\
\hline
\end{tabular}

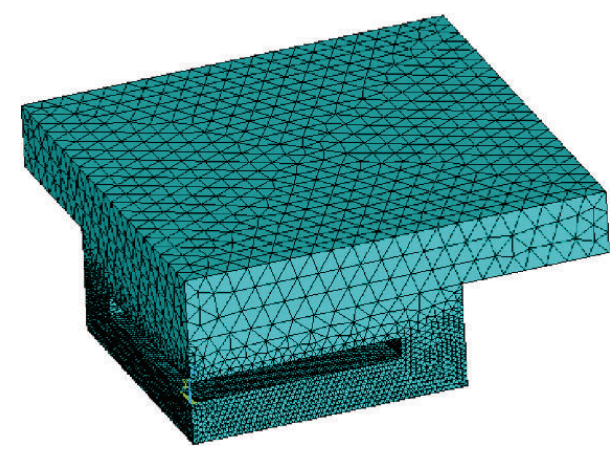

(a)

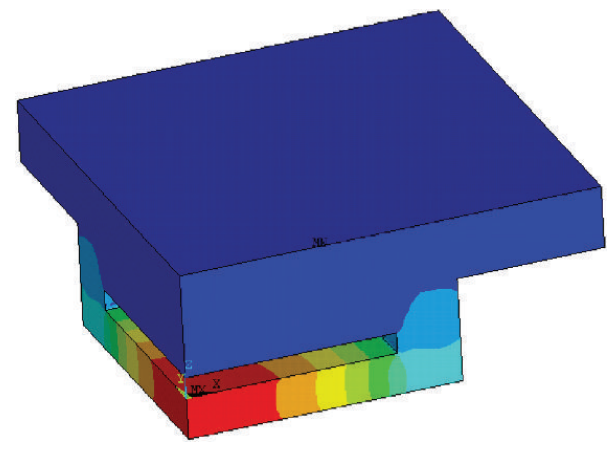

(b)

FIGURE 3: Simulated results in a constant velocity normal moving rectangular movable plate. (a) Typical mesh and (b) pressure distribution.

\section{Normal Motion with Oscillating Velocity}

4.1. Boundary Pressure. Consider the movable plate oscillating in air close to the fixed plate, the displacement of the movable plate is assumed to be a harmonic function of time $h(t)=H \sin (\omega t)$, where $H$ and $\omega$ are the amplitude and frequency of motion, respectively. The corresponding oscillating velocity of the movable plate is

$$
w(t)=\frac{\partial H}{\partial t}=H \omega \cos (\omega t) .
$$

The acoustic impedance of a slot of channel length $D_{p}$, cross section length $2(a+b)$, and cross section width $\delta$ is [10]

$$
Z_{A}=R_{A}+j \omega M_{A}=\frac{6 \mu D_{p}}{\delta^{3}(a+b)}+j \omega \frac{3 \rho_{a} D_{p}}{5 \delta(a+b)} .
$$

The equivalent circuit topology is shown in Figure 4. The equivalent values of the resistance and the inductance are given in (22). The venting volume of gas through the slot is equal to the reduced volume of gas under the plate, $Q=a b H \omega \cos (\omega t)$. The boundary pressure at the edge of the movable plant with oscillating velocity can be obtained as

$$
P_{\mathrm{BO}}=P_{c} \cos (\omega t)+P_{s} \sin (\omega t),
$$

where the coefficient $P_{c}$ and $P_{s}$ are

$$
P_{c}=\frac{6 \mu D_{P} a b H \omega}{\delta^{3}(a+b)}, \quad P_{s}=-\frac{3 \rho_{a} D_{P} a b H \omega^{2}}{5 \delta(a+b)} .
$$

4.2. Damping Force. Similarly, the linearized Reynolds equation of normal motion with oscillating velocity has the same form as (2). The effective source term in (5) is

$$
\rho(r, t)=-\frac{\alpha^{2} \eta}{4 \pi} \frac{\partial H}{\partial t}=-\frac{\alpha^{2} \eta}{4 \pi} \frac{H \omega}{h_{0}} \cos (\omega t) .
$$

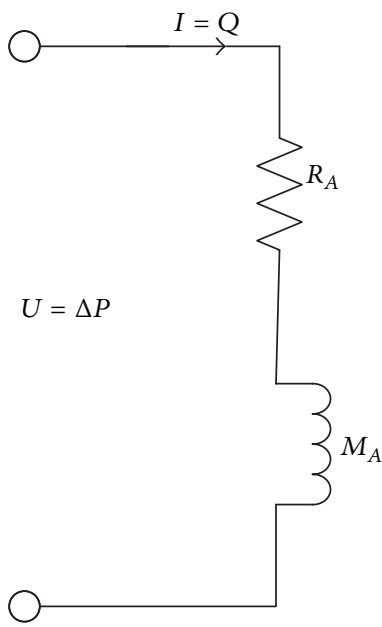

FIGURE 4: Electrical equivalent circuit of acoustic impedance.

The boundary pressures are not trivial which can be obtained by (23)

$$
\phi=P\left( \pm \frac{a}{2}, Y, t\right)=P\left(X, \pm \frac{b}{2}, t\right)=P_{\mathrm{BO}} .
$$

The first term of (10) representing the effect of volume sources is

$$
\begin{aligned}
P_{1}(r, t) & =\int_{0}^{t^{+}} d t^{\prime} \int d V^{\prime} \rho\left(r^{\prime}, t^{\prime}\right) G\left(r, t \mid r^{\prime}, t^{\prime}\right) \\
& =\sum_{m, n=\text { odd }} \frac{8(-1)^{(m+n) / 2}}{m n \pi^{2}} \cdot \frac{H \eta \omega}{H_{0}}
\end{aligned}
$$




$$
\begin{aligned}
& \frac{2 \cos (\omega t) \lambda_{m n}^{2} / \alpha^{2}+2 \omega \sin (\omega t)}{\left(\lambda_{m n}^{2} / \alpha^{2}\right)^{2}+\omega^{2}} \\
& \cdot \cos \frac{m \pi x}{a} \cos \frac{n \pi y}{b} .
\end{aligned}
$$

The resultant force under ideal vented conditions is the integration of the pressure variation across the rectangular plate area:

$$
\begin{aligned}
& F_{1}(t) \\
& =\sum_{m, n=\text { odd }} \frac{64}{m^{2} n^{2} \pi^{4}} \\
& +\sum_{m, n=\text { odd }} \frac{\frac{\eta \sigma P_{a} a b}{\omega H_{0}} \frac{\left(m^{2} \pi^{2}+\left(a^{2} / b^{2}\right) n^{2} \pi^{2}\right)}{\left(m^{2} \pi^{2}+\left(a^{2} / b^{2}\right) n^{2} \pi^{2}\right)^{2}+\sigma^{2}} H \omega \cos (\omega t)}{H_{0}} \\
& \times \frac{\eta \sigma^{2} P_{a} a b}{\left(m^{2} \pi^{2}+\left(a^{2} / b^{2}\right) n^{2} \pi^{2}\right)^{2}+\sigma^{2}} H \sin (\omega t),
\end{aligned}
$$

where $\sigma$ is the squeeze number, $\sigma=\omega a^{2} \alpha^{2}=12 \mu \omega a^{2} / h_{0}^{2} P_{a}$. The resultant force has two components: the spring force, which is in phase with the displacement, and damping force, which is in phase with the velocity. These results, consistent with those of Andrews et al. [3], are under ideally vented conditions.

The second term of (10) represents the border effect. The pressure distribution on the movable plate caused by nontrivial boundary pressure is

$$
\begin{aligned}
& P_{2}(r, t) \\
& =-\frac{1}{4 \pi} \int_{0}^{t^{+}} d t^{\prime} \int d S^{\prime} \phi \frac{\partial G}{\partial n} \\
& =-\sum_{m, n=\mathrm{odd}}\left[\left(\frac{P_{c} \lambda_{m n}^{2}}{\alpha^{2}}-P_{s} \omega\right) \cos (\omega t)\right. \\
& \left.+\left(\frac{P_{s} \lambda_{m n}^{2}}{\alpha^{2}}+P_{c} \omega\right) \sin (\omega t)\right) \\
& \left.\left.\times\left(\frac{\lambda_{m n}^{2}}{\alpha^{2}}\right)^{2}+\omega^{2}\right)^{-1}\right] \\
& \cdot(-1) \frac{m+n}{2} \frac{16}{\alpha^{2}} \frac{\lambda_{m n}^{2}}{m n \pi^{2}} \cdot \cos \frac{m \pi x}{a} \cos \frac{n \pi y}{b} .
\end{aligned}
$$

Integrating over the area of the movable plate, the resultant force caused by nontrivial boundary pressure is

$$
\begin{aligned}
& F_{2}(t) \\
& =\frac{64 P_{a} a b}{\pi^{4}} \\
& \times \sum_{m, n=\text { odd }}\left(P_{c}\left(m^{2} \pi^{2}+\frac{a^{2}}{b^{2}} n^{2} \pi^{2}\right)^{2}-P_{s} \sigma\left(m^{2} \pi^{2}+\frac{a^{2}}{b^{2}} n^{2} \pi^{2}\right)\right) \\
& \times\left(\left(m^{2} \pi^{2}+\frac{a^{2}}{b^{2}} n^{2} \pi^{2}\right)^{2}+\sigma^{2}\right)^{-1} \\
& \cdot \frac{\cos (\omega \mathrm{t})}{m^{2} n^{2}}+\frac{64 P_{a} a b}{\pi^{4}} \\
& \times \sum_{m, n=\text { odd }}\left(P_{s}\left(m^{2} \pi^{2}+\frac{a^{2}}{b^{2}} n^{2} \pi^{2}\right)^{2}+P_{c} \sigma\left(m^{2} \pi^{2}+\frac{a^{2}}{b^{2}} n^{2} \pi^{2}\right)\right) \\
& \times\left(\left(m^{2} \pi^{2}+\frac{a^{2}}{b^{2}} n^{2} \pi^{2}\right)^{2}+\sigma^{2}\right)^{-1} \cdot \frac{\sin (\omega t)}{m^{2} n^{2}} .
\end{aligned}
$$

Accordingly, the total resultant force is

$$
F(t)=F_{1}(t)+F_{2}(t)
$$

This total resultant force is also caused by the damping pressure distribution on the surface of the movable plate. Equation (31) represents the net reaction force for square plate with slotted vents along all four edges, which comprises two kinds of force: the air damping force and the air spring force.

4.3. Instance Analysis. As an example of this model, consider a rigid, square plate with motion normal to its surface and with slotted vents along all four edges through which the trapped gas film is vented. The square plate moves with an oscillating velocity. The normalized amplitude of the vibrating plate $H / H_{0}$ equals to 0.1 . The channel length of the venting slot $D_{P}$ is half of the width of the movable plate $a$. The spring and damping components of the reaction force under free-venting condition and under slot-venting condition are shown in Figure 5(a). As can be seen, the venting conditions at the border have a significant influence on the damping force and the spring force. The damping force and the spring force under slot-venting condition are all larger than that under ideally venting condition. The damping force and the spring force for different normalized widths of $\delta / a$ are plotted in Figure 5(b). The smaller the width of the slot is, the greater the damping force and the spring force are. With normalized width $\geq 0.3$, the contribution of border effect to the damping force and the spring force is $>10 \%$.

\section{Conclusions}

The squeeze film damping analytical models with trivial pressure boundary condition fails to give satisfactory results 


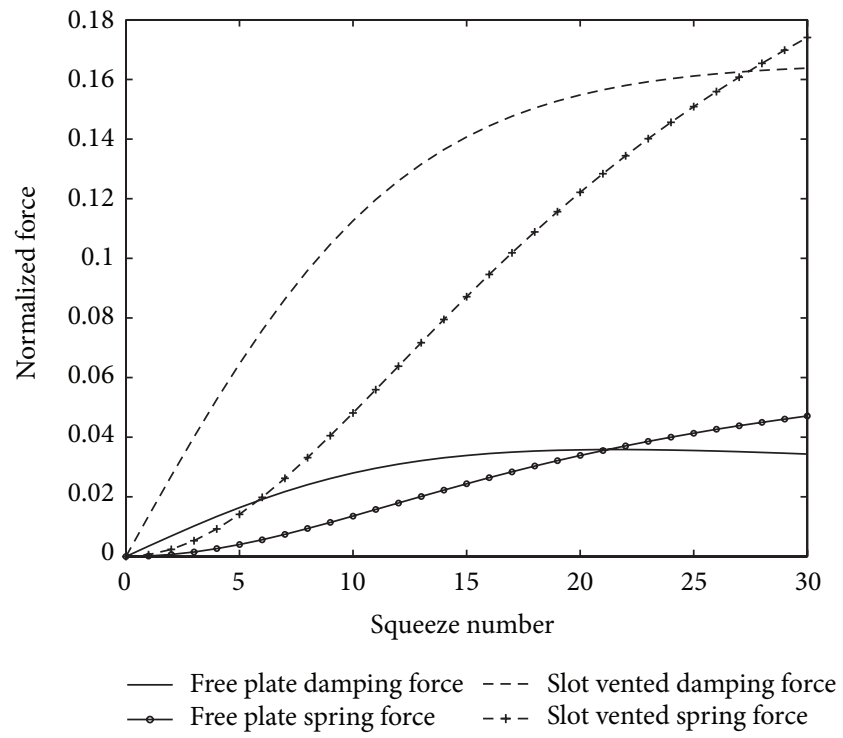

(a)

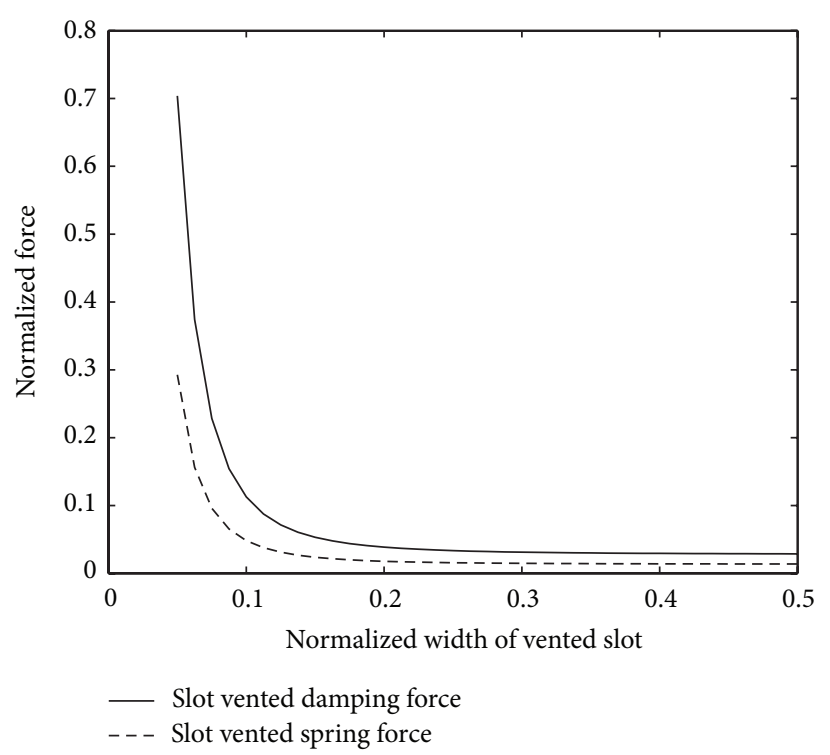

(b)

FIGURE 5: Result of an instance: (a) spring and damping forces (b) relationship of slot width and force.

for structures under nontrivial pressure boundary conditions such as the air venting gap between movable plate and sidewall being small. In order to consider the border effects in the analytically model, the nonlinear Reynolds equation is solved with inhomogeneous boundary conditions using Green's function approach in present paper. Analytical results in a range of MEMS devices with different structural parameters are compared with the CFD numerical simulation results. Moreover, the contribution of border effect for the damping force and the spring force is analyzed.

\section{Conflict of Interests}

The authors declare that there is no conflict of interests regarding the publication of this paper.

\section{Acknowledgment}

This work is supported by 111 Project of B14042.

\section{References}

[1] M. Bao and H. Yang, "Squeeze film air damping in MEMS," Sensors and Actuators A: Physical, vol. 136, no. 1, pp. 3-27, 2007.

[2] G. Schrag and G. Wachutka, "Physically based modeling of squeeze film damping by mixed-level system simulation," Sensors and Actuators A: Physical, vol. 97-98, pp. 193-200, 2002.

[3] M. Andrews, I. Harris, and G. Turner, "A comparison of squeeze-film theory with measurements on a microstructure," Sensors and Actuators A: Physical, vol. 36, no. 1, pp. 79-87, 1993.

[4] F. Pan, J. Kubby, E. Peeters, A. T. Tran, and S. Mukherjee, "Squeeze film damping effect on the dynamic response of a MEMS torsion mirror," Journal of Micromechanics and Microengineering, vol. 8, no. 3, pp. 200-208, 1998.
[5] A. Minikes, I. Bucher, and G. Avivi, "Damping of a microresonator torsion mirror in rarefied gas ambient," Journal of Micromechanics and Microengineering, vol. 15, no. 9, pp. 17621769, 2005.

[6] A. K. Pandey, R. Pratap, and F. S. Chau, "Analytical solution of the modified Reynolds equation for squeeze film damping in perforated MEMS structures," Sensors and Actuators A: Physical, vol. 135, no. 2, pp. 839-848, 2007.

[7] S. S. Mohite, H. Kesari, V. R. Sonti, and R. Pratap, "Analytical solutions for the stiffness and damping coefficients of squeeze films in MEMS devices with perforated back plates," Journal of Micromechanics and Microengineering, vol. 15, no. 11, pp. 20832092, 2005.

[8] Z. Hao, R. Clark, J. Hammer, M. Whitley, and B. Wingfield, "Modeling air-damping effect in a bulk micromachined 2D tilt mirror," Sensors and Actuators A: Physical, vol. 102, no. 1-2, pp. 42-48, 2002.

[9] T. Veijola, A. Pursula, and P. Råback, "Extending the validity of squeezed-film damper models with elongations of surface dimensions," Journal of Micromechanics and Microengineering, vol. 15, no. 9, pp. 1624-1636, 2005.

[10] R. B. Darling, C. Hivick, and J. Xu, "Compact analytical modeling of squeeze film damping with arbitrary venting conditions using a Green's function approach," Sensors and Actuators A: Physical, vol. 70, no. 1-2, pp. 32-41, 1998.

[11] T. Veijola, K. Ruokonen, and I. Tittonen, "Compact model for the squeezed-film damping including the open border effects," in Proceedings of the 4th International Conference on Modeling and Simulation of Microsystems (MSM '01), pp. 76-79, Hilton Head Island, SC, USA, March 2001.

[12] P. K. Kundu and I. M. Cohen, Fluid Mechanice, Academic Press, Waltham, Mass, USA, 4th edition, 2012. 


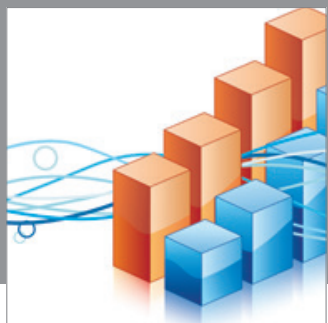

Advances in

Operations Research

mansans

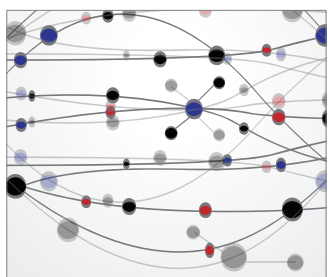

The Scientific World Journal
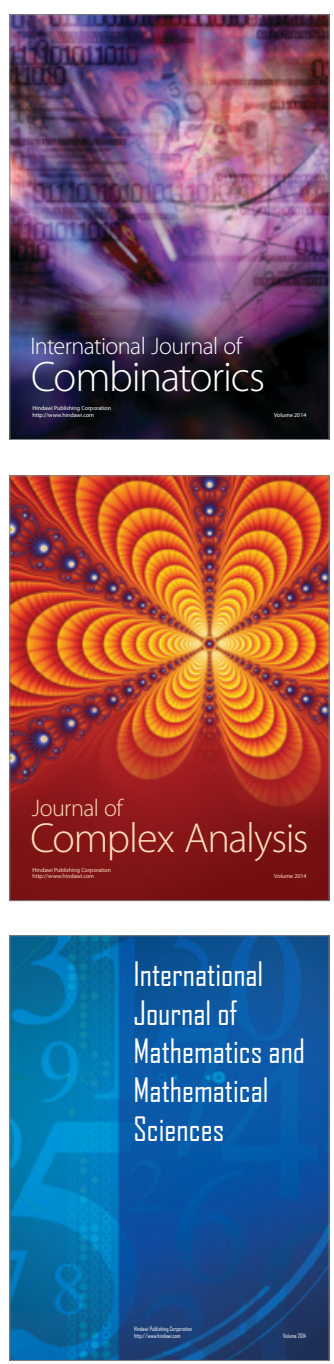
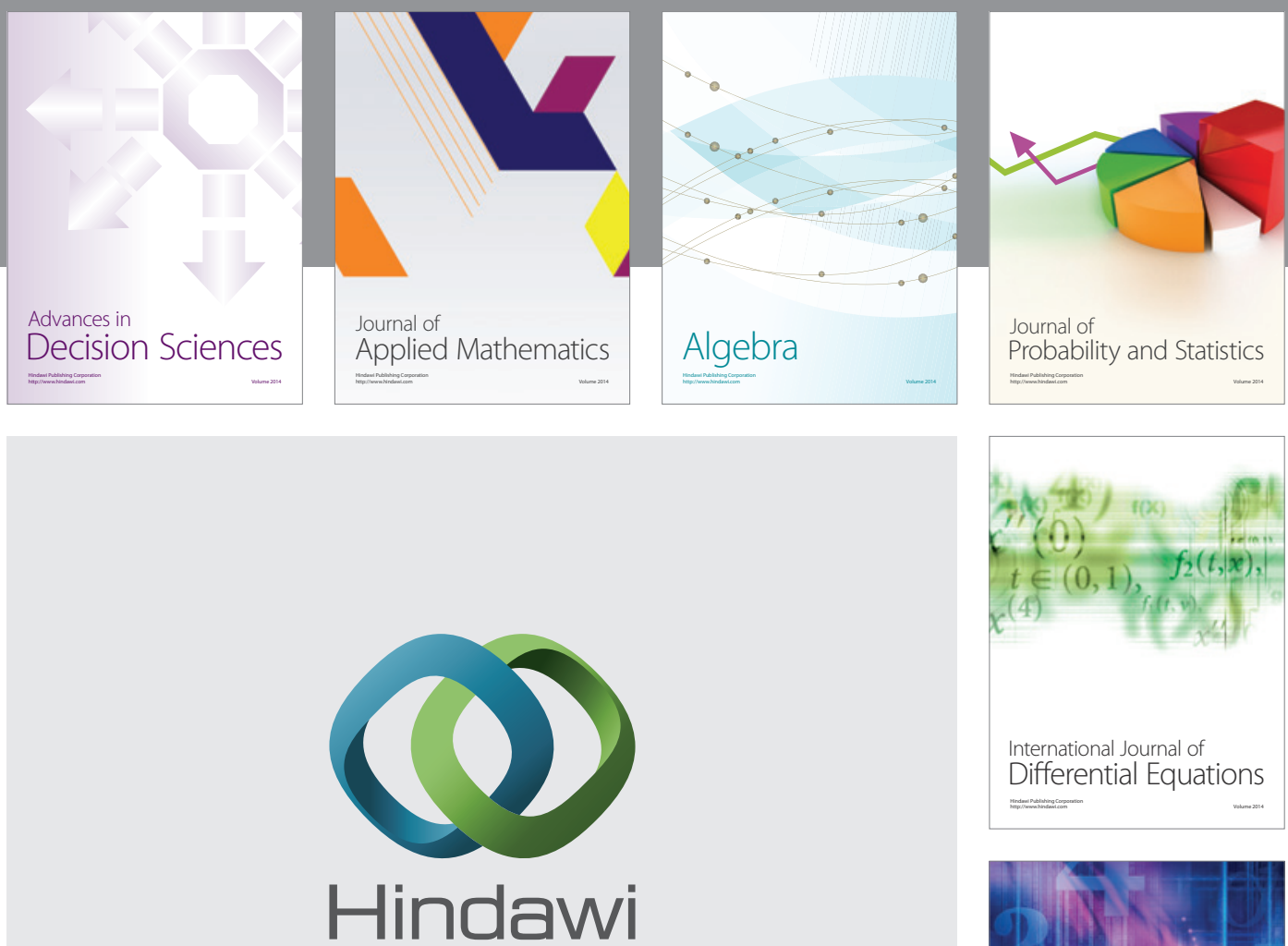

Submit your manuscripts at http://www.hindawi.com
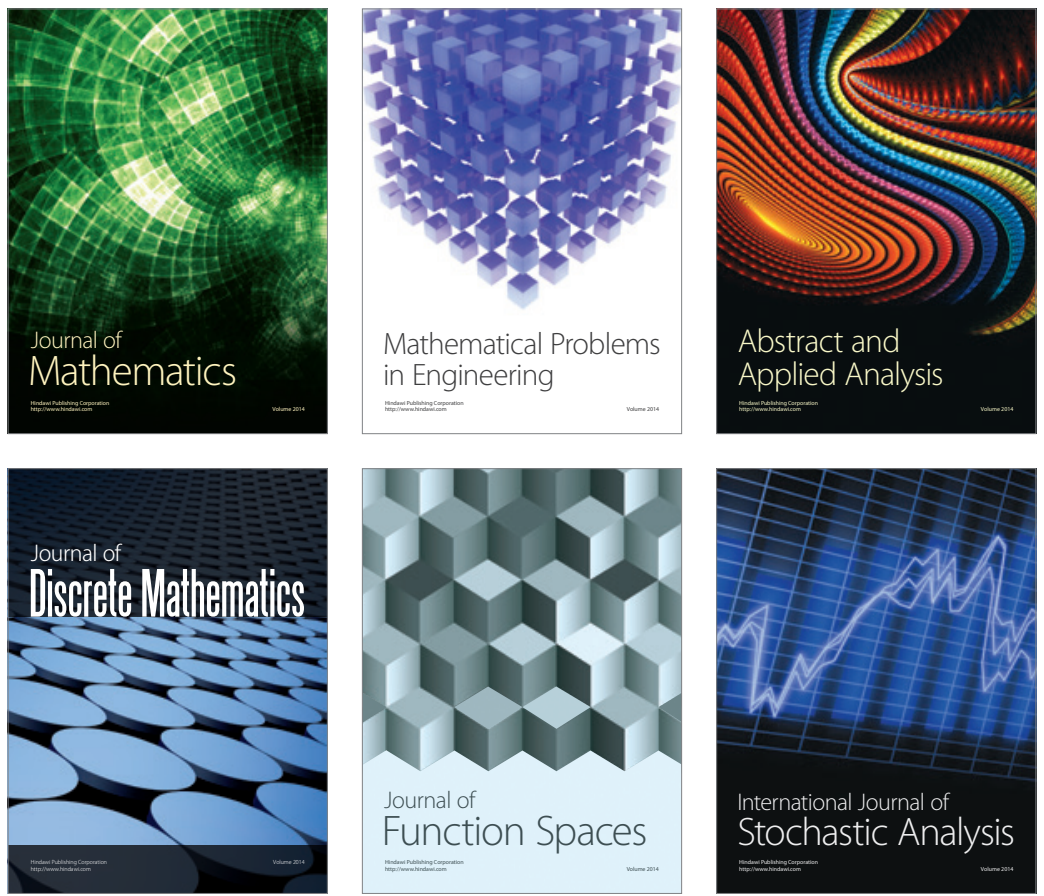

Journal of

Function Spaces

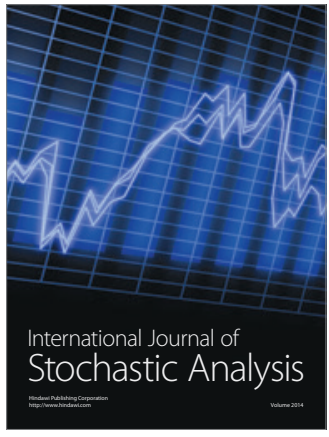

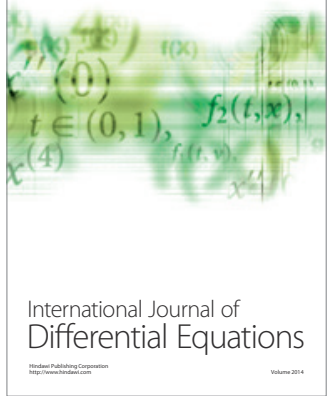
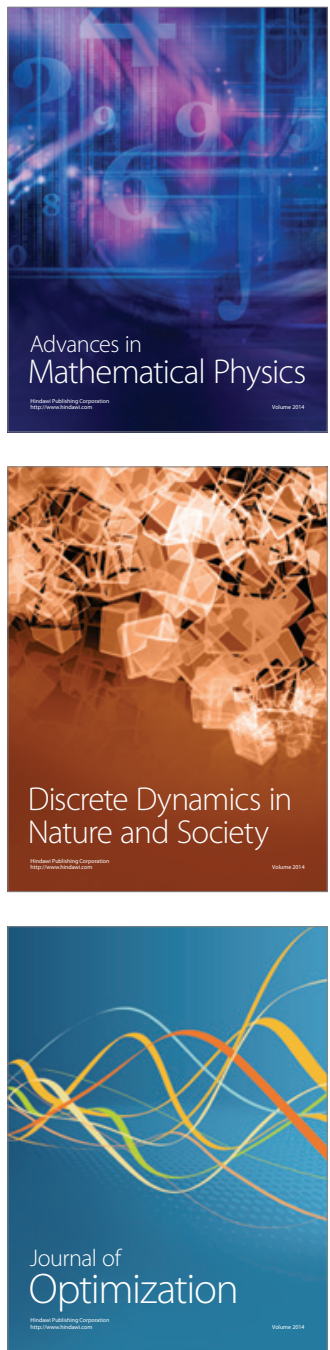